\title{
NORMAL VALUES FOR PHYSICAL PARAMETERS OF THE HEAD, FACE AND HAND IN JAPANESE CHILDREN
}

\author{
Mie IGarashi and Tadashi KaJII \\ Department of Pediatrics, Yamaguchi University \\ School of Medicine, Ube, Japan 755
}

\begin{abstract}
Summary A total of 19 physical parameters of the head, face and hand were measured in 1,800 Japanese children of ages one month to 15 years. They were grouped according to sex and age: 1-3 month, 4-6 month, and 7-12 month age intervals for the first year, and thereafter yearly intervals. Exceptional values were excluded referring to the estimated mean and standard deviation. The results were presented in graphic forms with the mean value \pm 2 standard deviations (SD) for each sex and age group, and compared with the norms for Caucasian children.
\end{abstract}

\section{INTRODUCTION}

The norms for physical measurements in today's Japanese children are largely unavailable. The weight, length (height) and head circumference of children ranging from infants to 6-year-olds are updated every ten years based on a nationwide survey, the most recent being the one carried out in 1980 (Mothers' \& Children's Health \& Welfare Association, 1981). The weight, height, sitting height and chest circumference for 5- to 17-year-olds in 1980 are also available (Resarch Statistics Division, Minister's Secretariat, The Ministry of Education, Science and Culture, 1982). Norms for the inner canthal distance have been published (Suzuki et al., 1977). These are a few exceptions.

In view of the dearth of information, we carried out measurements of the physical parameters of the head, face and hand of normal Japanese children.

\section{MATERIALS AND METHODS}

The study population consisted of 1,800 normal Japanese children of both sexes, 900 boys and 900 girls, aged one month to 15 years. The infants and children

Received January 7, 1988; Accepted January 26, 1988

Offprint request to: Dr. Mie Igarashi, Department of Pediatrics, Yamaguchi University School of Medicine, Ube, Yamaguchi 755, Japan 
up to 5 years of age were drawn from infants who visited the outpatient clinic of Yamaguchi University Hospital and that of Onoda Municipal Hospital for regular physical check-ups, and from children who attended nurseries and kindergartens in Ube city. Children of 6 to 15 years of age were drawn from a primary school and two junior high schools in Ube city. The study was carried out during the period from September, 1983 to March, 1984.

The infants and children were grouped according to sex and age: 1-3, 4-6, and 7-12 month-old groups for children under one year old, and groups of yearly intervals thereafter for children from 2 to 15 years-old. Each sex and age group thus consisted of 50 children.

A set of anthropometric instruments (Koshi Co., Japan) were used throughout the study. The set included sliding calipers, a spreading caliper, a goniometer and a measuring tape. An instrument made from X-ray film with parallel reference lines drawn on it was used in the determination of the position and size of the ear (Feingold

Table 1. Parameters of measurements.

1. Head circumference: the measurement of the circumference at the glabella anteriorly and at the maximum point of the occiput posteriorly.

2. Head length: the distance from the glabella to the prominence of the occiput.

3. Head breadth: maximum biparietal distance.

4. Total head height: vertical distance from the vertex to the menton.

5. Face height: the distance from the nasion to menton with the teeth in occlusion.

6. Face breadth: the maximum distance between the zygomas as measured by calipers.

7. Inner canthal distance: the distance between the inner angles of the palpebral fissures.

8. Outer canthal distance: the distance between the outer angles of the palpebral fissures.

9. Interpupillary distance: the distance between the centers of both pupils with the eyes looking straight ahead.

10. Nose length: the distance from the nasion to the subnasion.

11. Nose breadth: the distance between the most lateral aspects of the alae nasi.

12. Philtrum length: the distance from the base of the columella to the midline depression of the vermilion border.

13. Mouth breadth: interangular distance of the mouth at rest.

14. Upper lip thickness: the distance at the midline from the vermilion border of the upper lip to the aperture.

15. Lower lip thickness: the distance at the midline from the vermilion border of the lower lip to the aperture.

16. Ear length: the standard line binding the inner canthus and the occipital prominence, using the device described in Fig. 2. The sum of the vertical distances from this line to the superior and inferior aspects of the left ear.

17. Palm length: the distance from the distal flexion crease of the wrist to the proximal flexion crease of the right middle finger.

18. Middle finger length: the distance from the proximal flexion crease to the tip of the right middle finger.

19. Fifth finger length: the distance from the proximal flexion crease to the tip of the right fifth finger. 
and Bossert, 1974). Measurements were performed for 19 physical parameters of the head, face and hand as listed in Table 1., Figs. 1 and 2). All measurements were carried out by one of the authors (M.I.) and recorded by an assistant.

The data thus obtained were analyzed by computer programs written in Microsoft Multiplan using an NEC 9801 personal computer. The programs used the raw data to calculate the various parameters studied and then calculate the mean, standard deviation (SD), and minimum and maximum values of each observation. Exceptional, outlying values were rejected referring to the expected extreme values for a normal distribution of the estimated mean and standard deviation. The expected extreme values were calculated at the $1 \%$ level, referring to Smirnoff's constant. Thus, of the 34,200 measurements, $150(0.4 \%)$ were disregarded. The mean value and \pm 2 standard deviations were then calculated for each category. There were chance fluctuations in the mean values and \pm 2 standard deviations, and they were smoothed by eye.
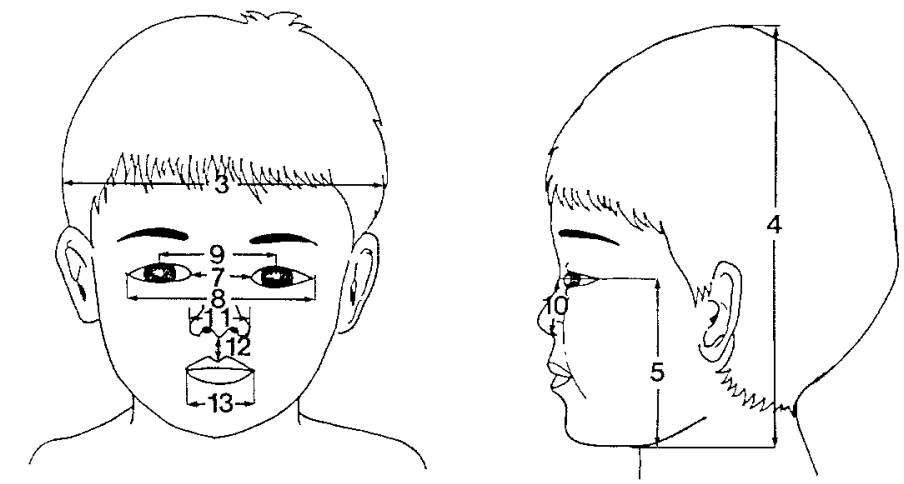

Fig. 1. Physical parameters of the head and face. 3, head breadth; 4, total head height; 5 , face height; 7 , inner canthal distance; 8 , outer canthal distance; 9 , interpupillary distance; 11 , nose breadth; 12 , philtrum length; 13 , mouth breadth.
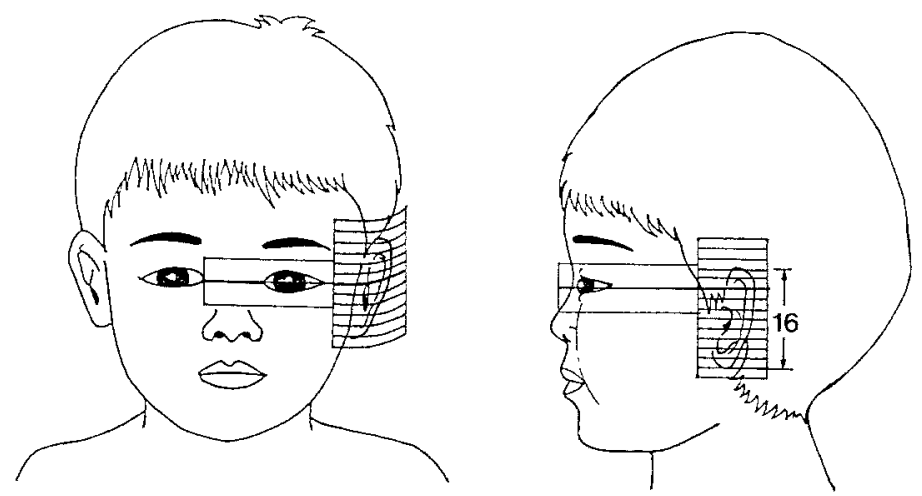

Fig. 2. Ear length. 


\section{RESULTS}

The results were presented in graphic forms with the mean \pm 2 standard deviations (SD) for each parameter, sex and age group (Graphs 1-19). The mean values for Japanese children (solid lines) were compared with those for Caucasian children (broken lines). The latter included parameter 1 (Nellhaus, 1968), parameters 2, 3, 5 and 6 (Pryor, 1966), parameters 7-9, 17 and 18 (Feingold and Bossert, 1974), parameter 12 (personal communication from Feingold, M., quoted by Goodman and Gorlin, 1977) and parameter 13 (Cervenka et al., 1969).

\section{DISCUSSION}

The significance of setting norms for physical parameters in Japanese children is obvious. Dysmorphic features have often been based on subjective impression. A child with Down syndrome often gives the false impression of hypertelorism due to its epicanthal folds and flat nasal bridge, but in fact its intercanthal distance is not beyond the normal range (Suzuki et al., 1977).

Measuring physical parameters is simple, quick, harmless and inexpensive. Minor anomalies may also occur in normal individuals, but they are often related to dysmorphic syndromes. The normal values we have described will provide a basis to better define new and old syndromes.

Acknowledgments We with to thank Miss H. Kawai for her assistance, Dr. I. Murano for his aid in computer analysis, and Drs. J. Okamoto and M. Tsukahara for their advice. Our thanks are also due to the help of the personnels of the hospitals, nurseries and kindergartens, and the teachers of the schools where the measurements were carried out.

\section{REFERENCES}

Cervenka, J., Figalova, P., and Gorlin, R.J. 1969. Oral intercommissural distance in children. Am. J. Dis. Child. 117: 434-435.

Feingold, M. and Bossert, W.H. 1974. Normal values for selected physical parameters: an aid to syndrome delineation. Birth Defects Orig. Art. Ser. 10 (13): 1-17.

Goodman, R.M. and Gorlin, R.J. 1977. Head and facial measurements. In Atlas of the Face in Genetic Disorders, Goodman, R.M. and Gorlin, R.J., eds., C.V. Mosby, Saint Louis, pp. 48-63.

Mothers' \& Children's Health \& Welfare Association, 1981. The Results of Survey on the Physical Growth and Development of Infants and Preschool Children, 1980. Compiled under the Supervision of Maternal \& Child Health Division, Children \& Families Bureau, Ministry of Health and Welfare.

Nellhaus, G. 1968. Head circumference from birth to eighteen years. Practical composite international and interracial graphs. Pediatrics 41 : 106-114.

Pryor, H.B. 1966. Charts of normal body measurements and revised width-weight tables in graphic form. J. Pediatr. 68: 615-631.

Research Statistics Division, Minister's Secretariat, The Ministry of Education, Science and Culture 1982. Report on School Health Survey, 1980, quated in J. Child Health 41: 244-245, 1982.

Suzuki, Y., Matsubara, T., and Toyohuku, T. 1977. Hypertelorism and its clinical significance. Shonika Rinsho (in Japanese) 30:26-30. 


\section{Head Circumference}
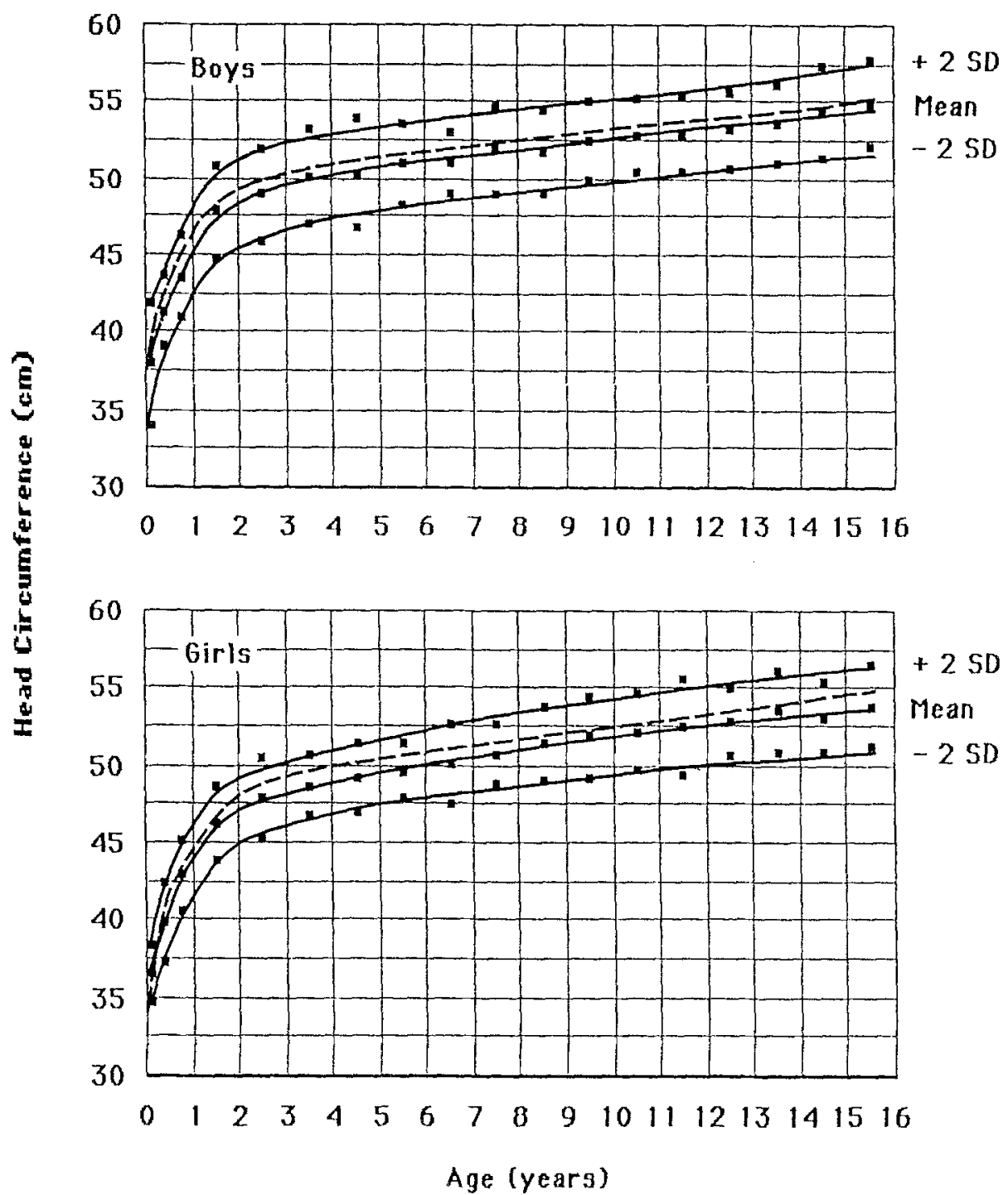

The head circumference in the children in our study was measured at the level of just above the eyebrows anteriorly and the maximum point of the occiput posteriorly. The measurement in the Caucasian children was performed at the level of the greatest frontal protuberance anteriorly and the occipital prominence posteriorly. This difference in measurement apparently accounted for the difference of the mean head circumference between Japanese and Caucasian children. 


\section{Head Length}

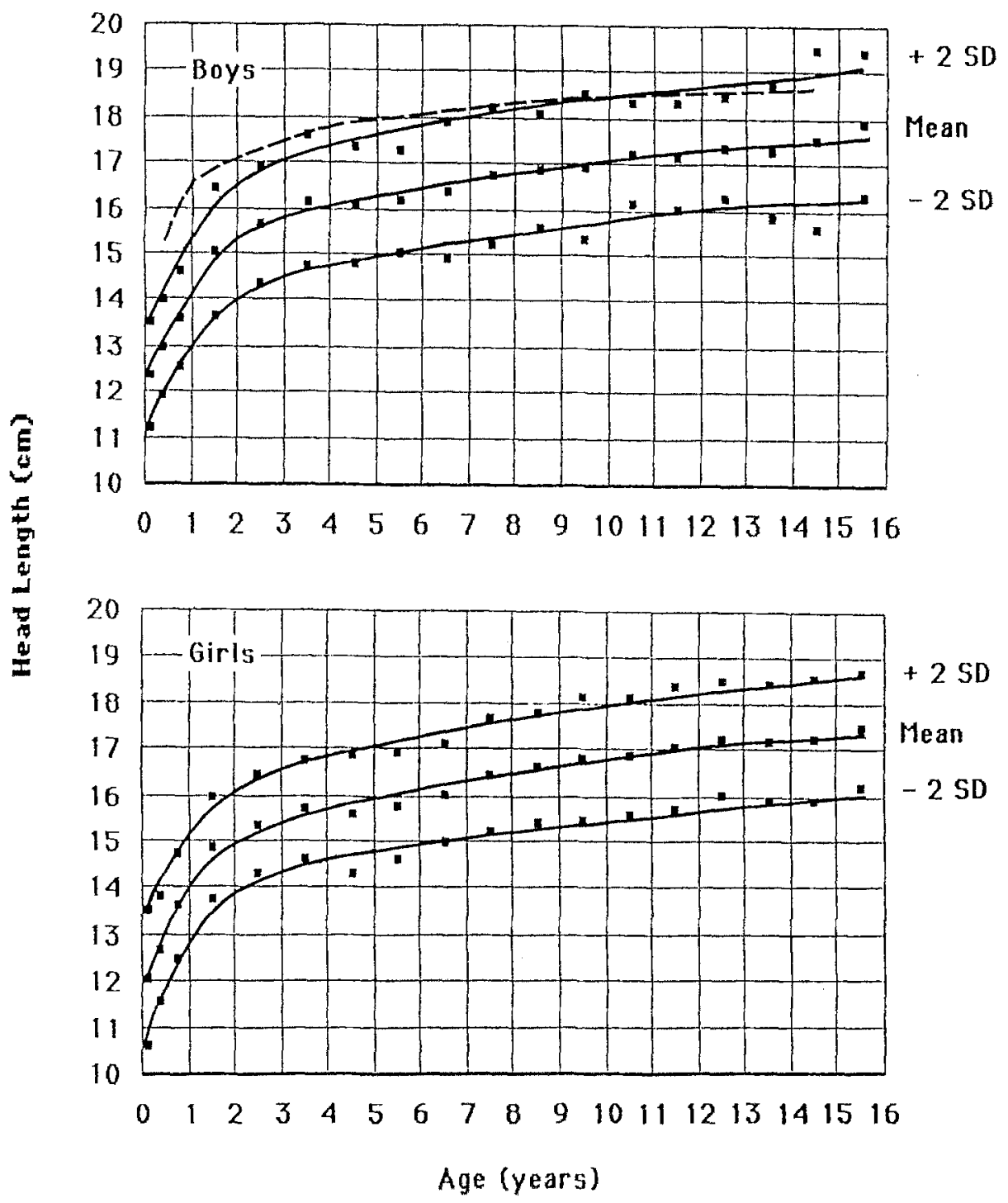




\section{Head Breadth}

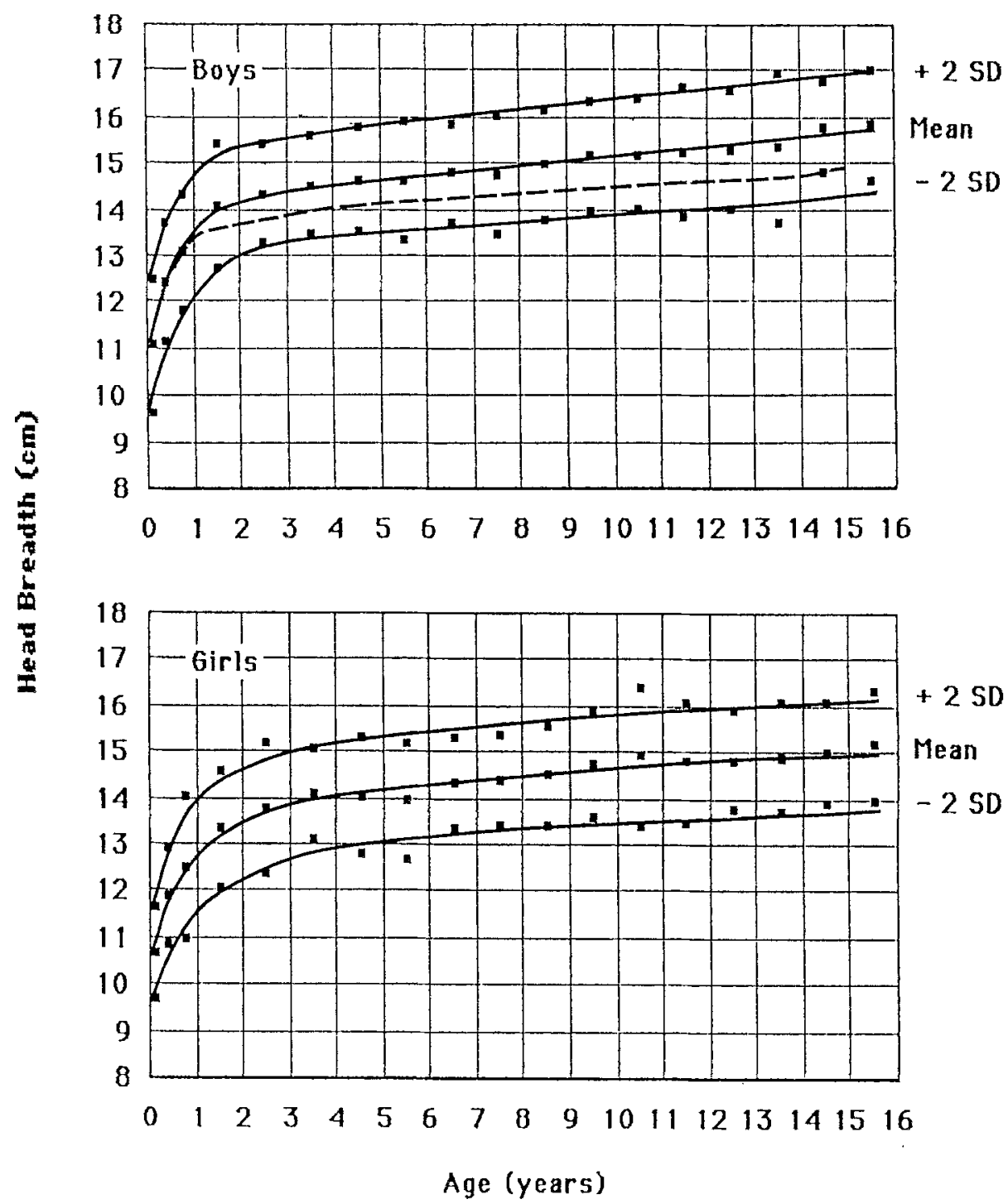




\section{Total Head Height}

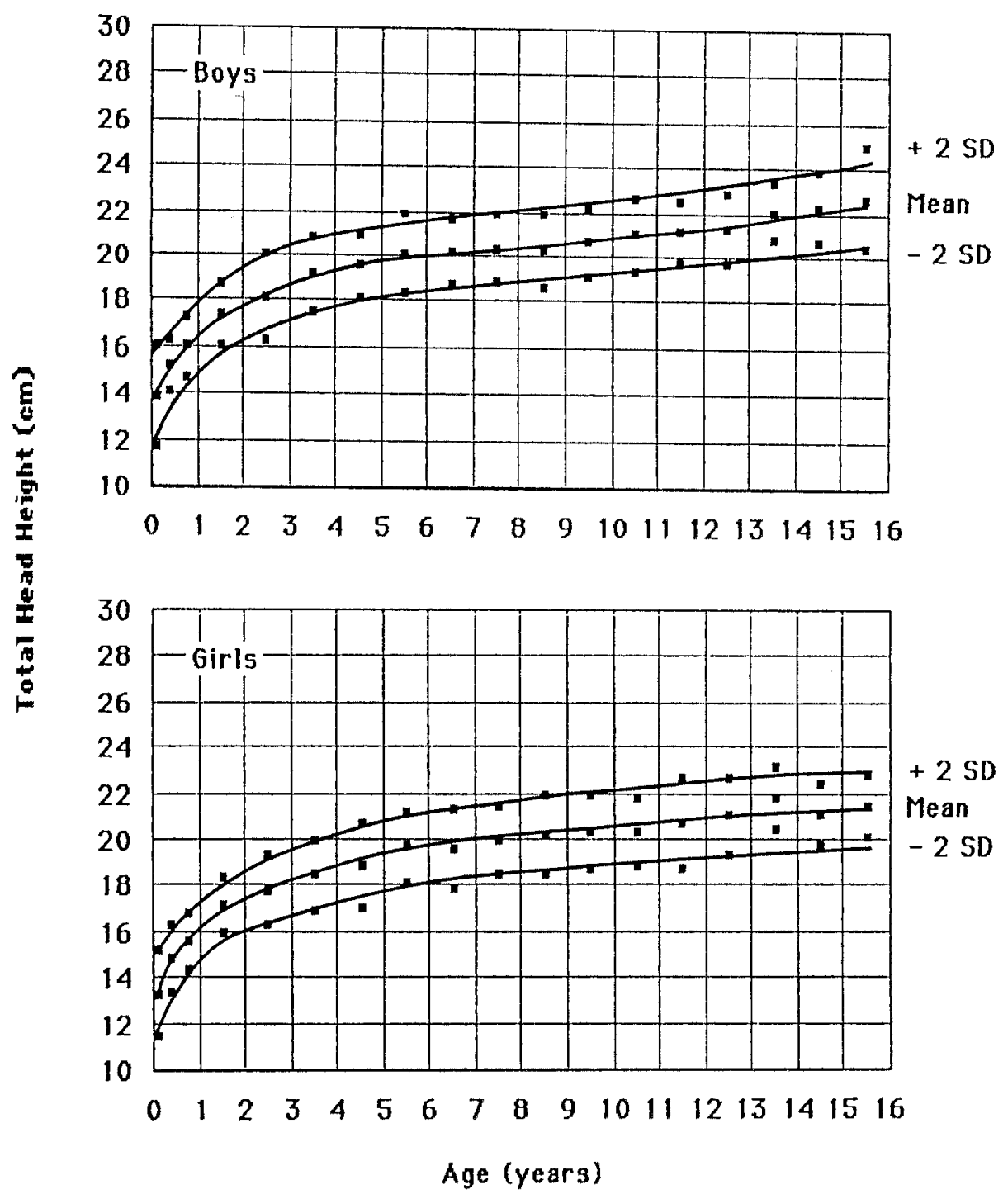




\section{Face Height}

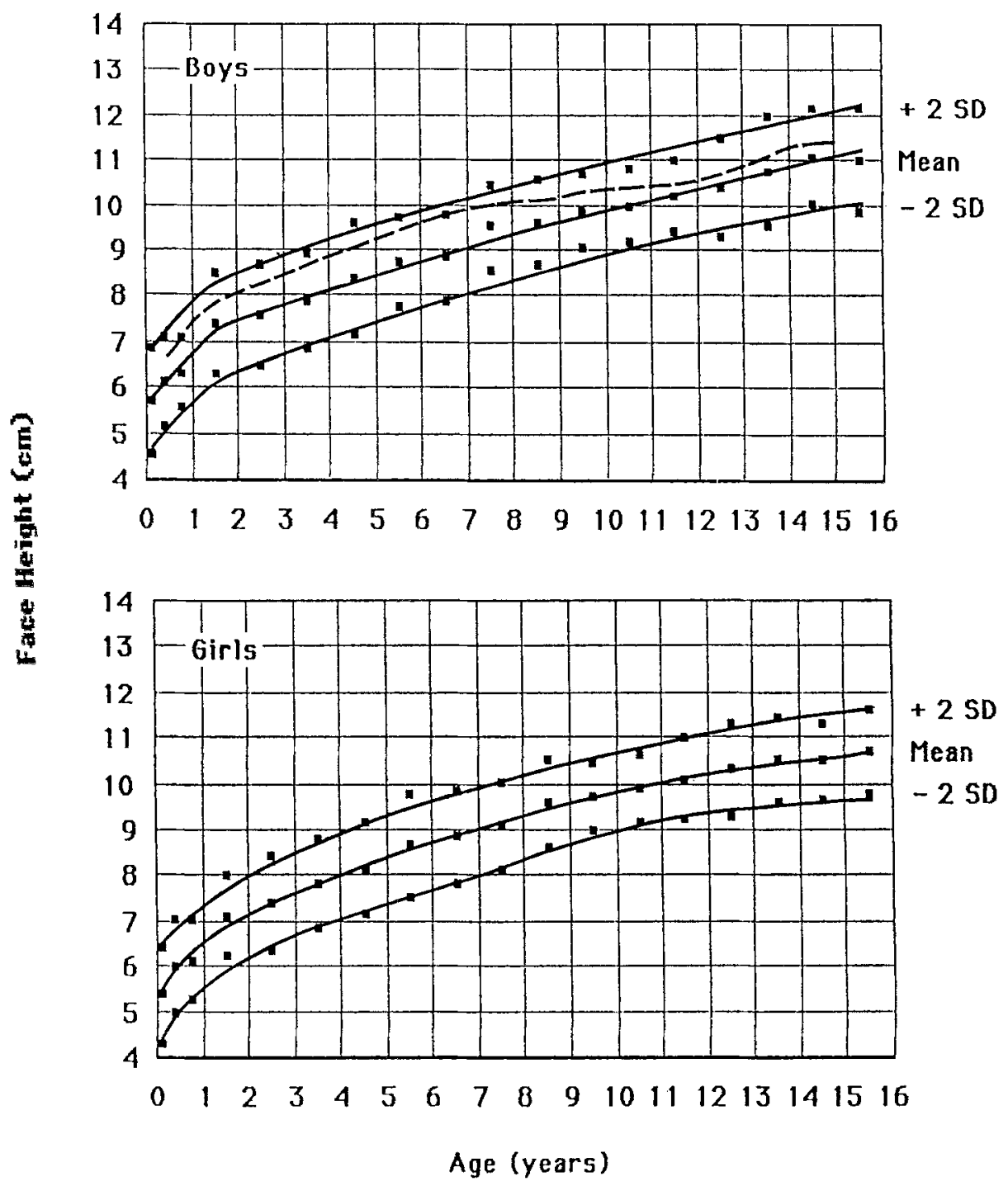




\section{Face Breadth}

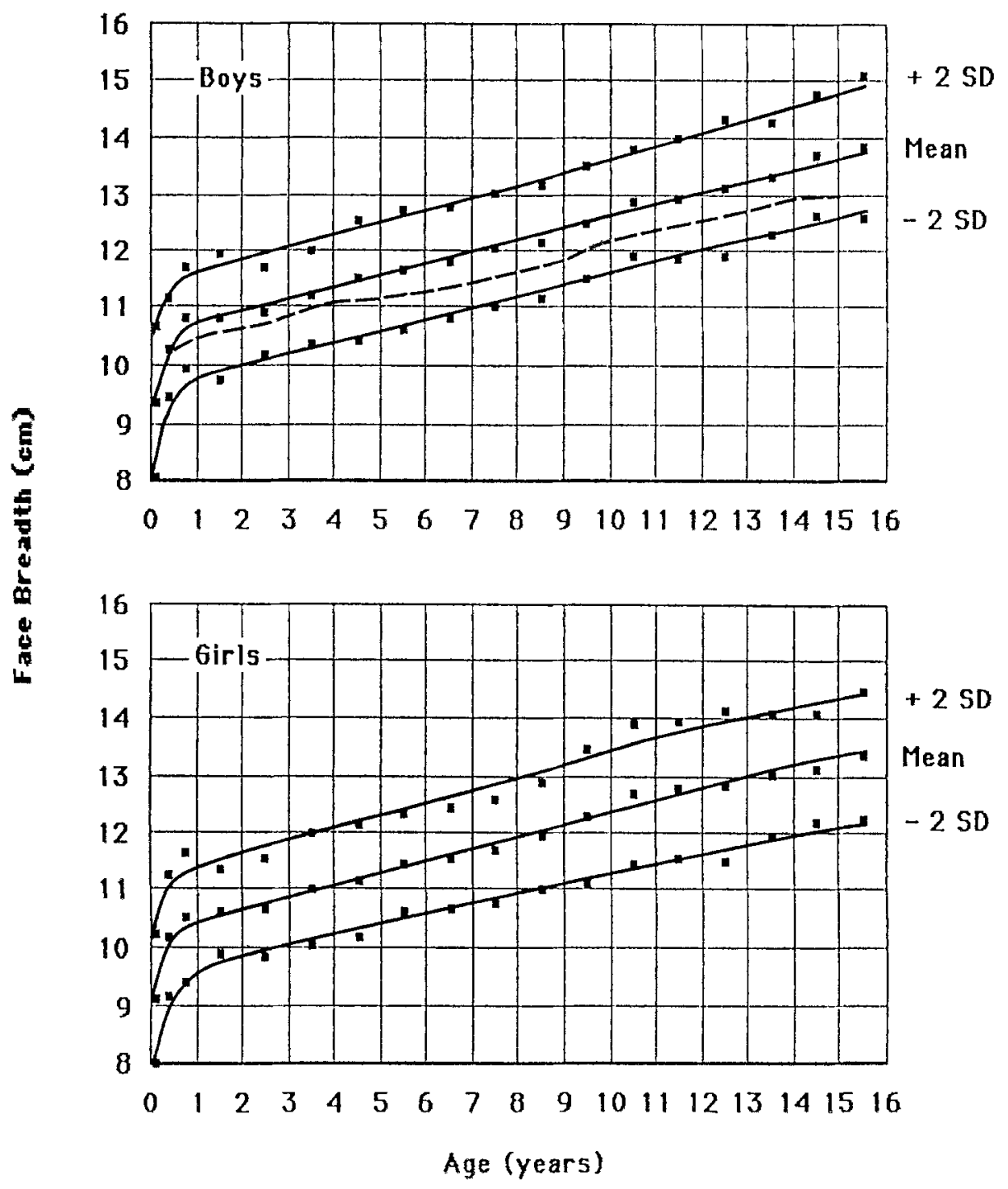




\section{Inner Canthal Distance}
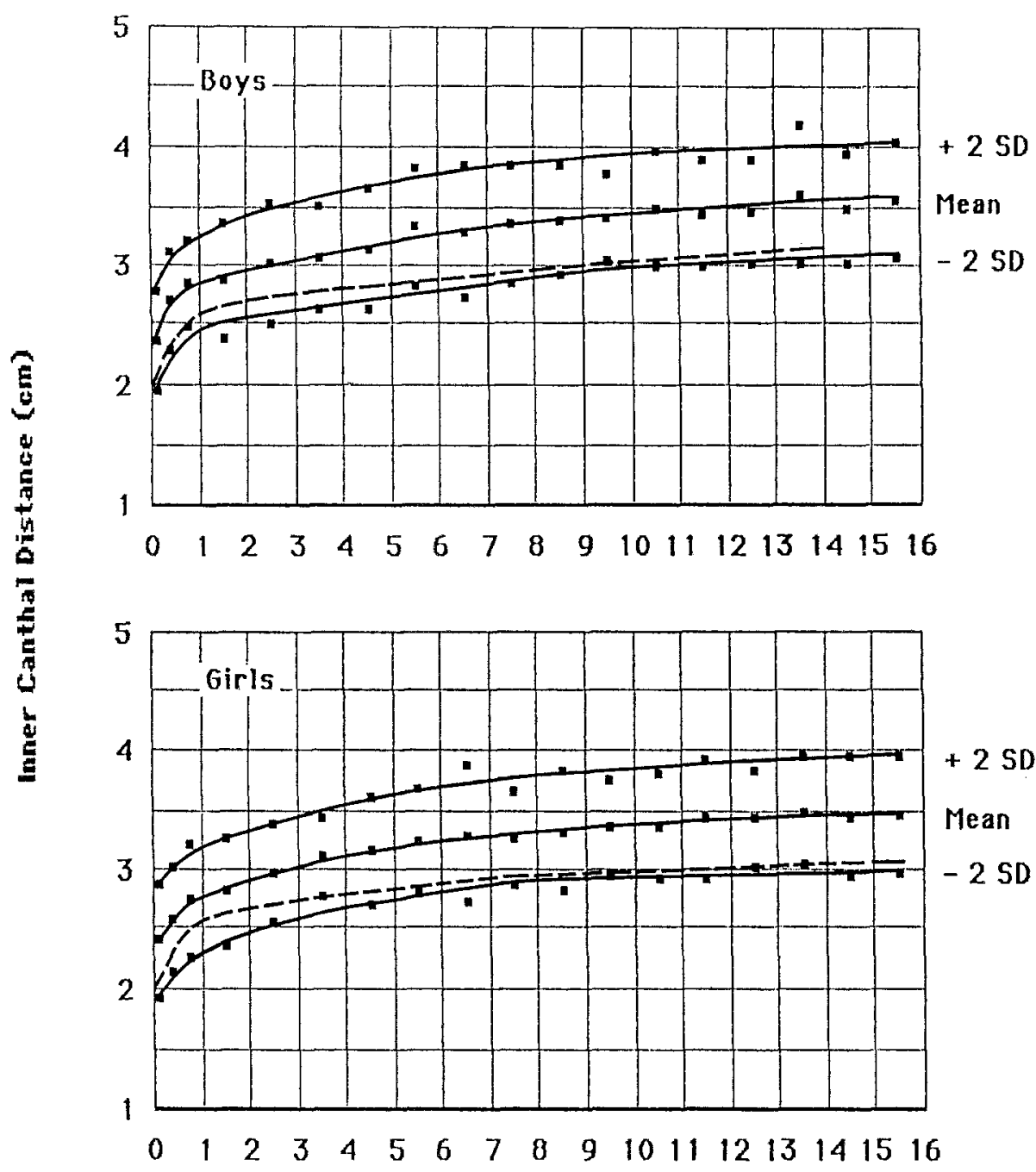

Age (years) 


\section{Duter Canthol Distance}

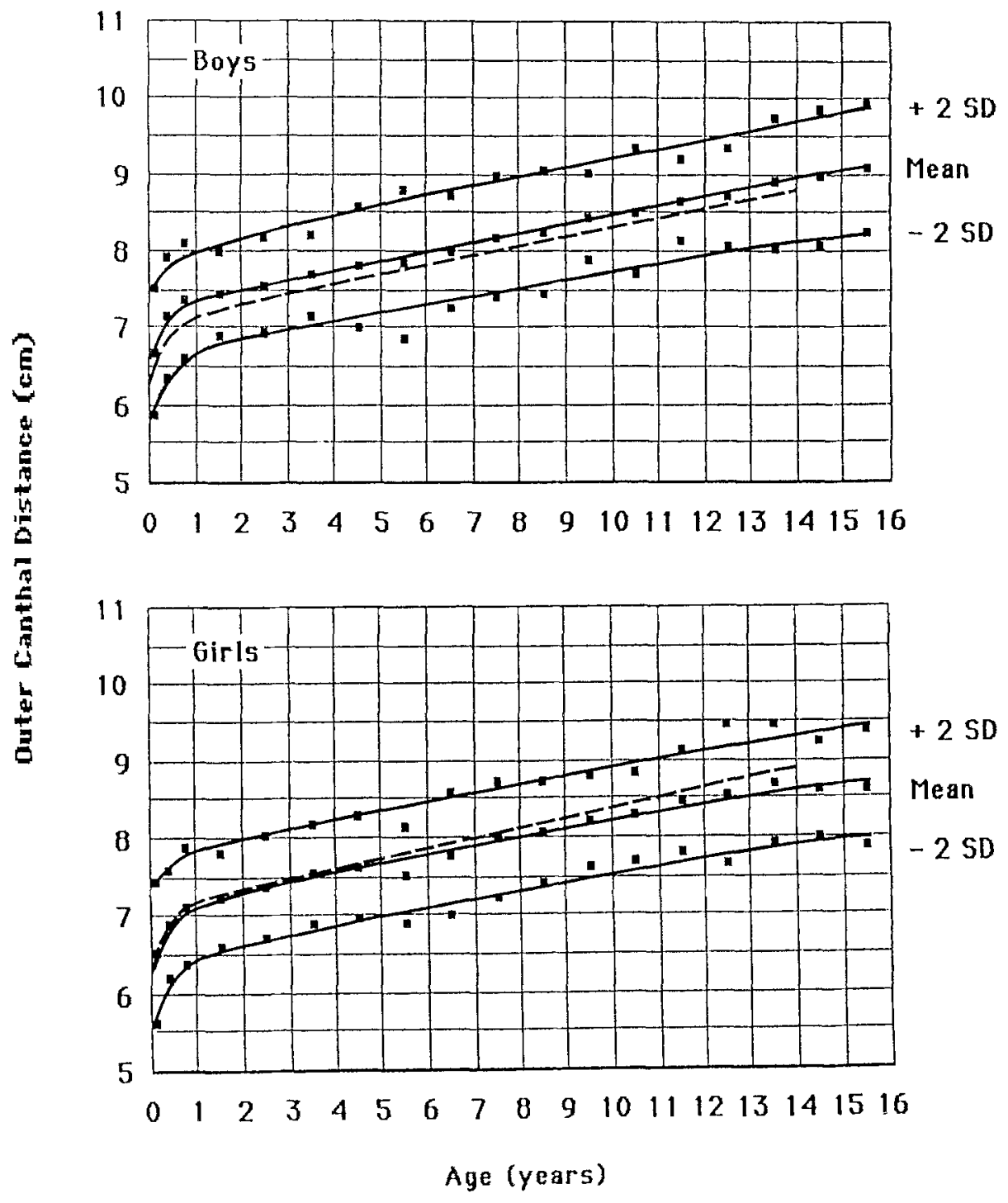




\section{Interpupillary Distance}

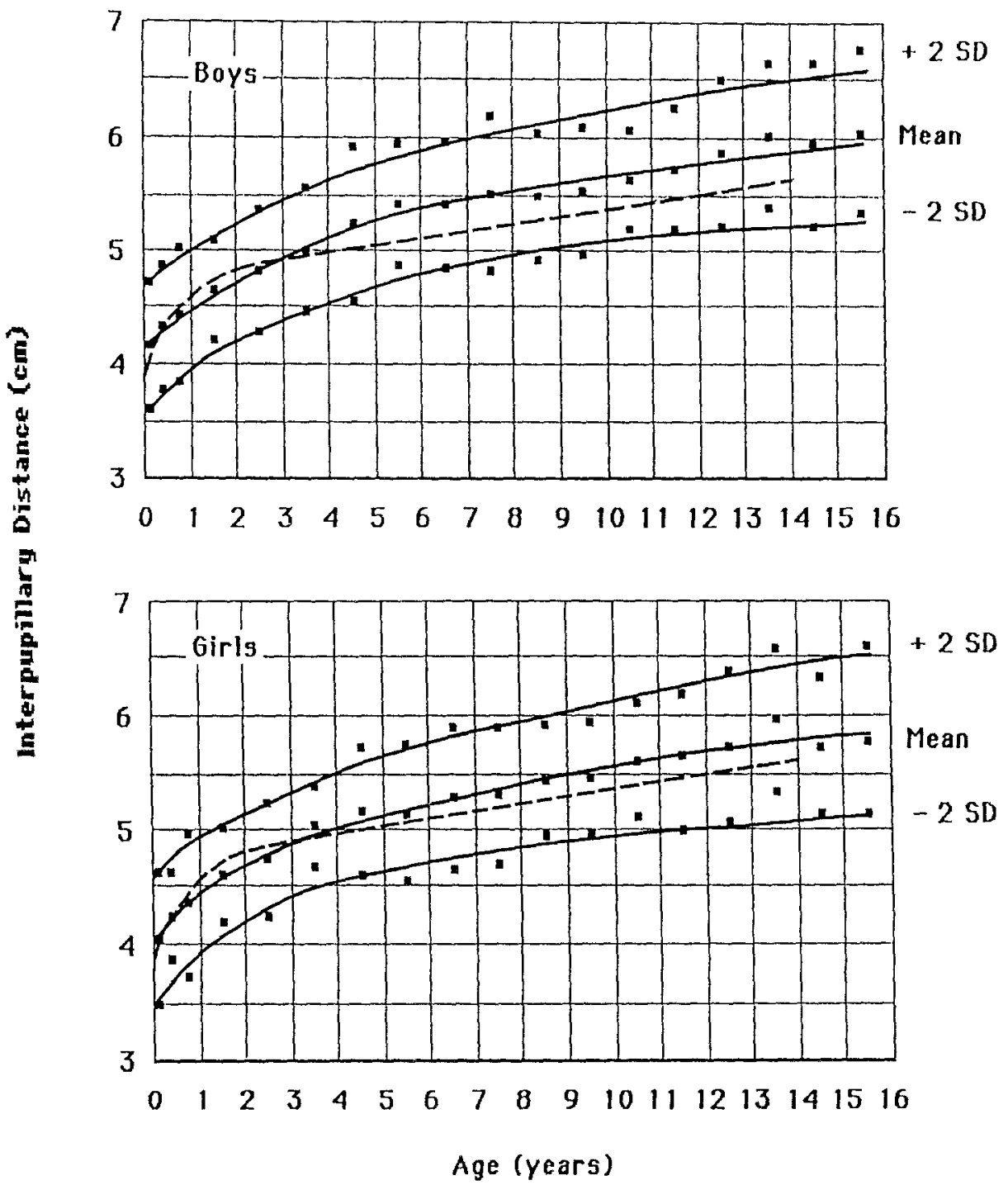




\section{Nose Length}

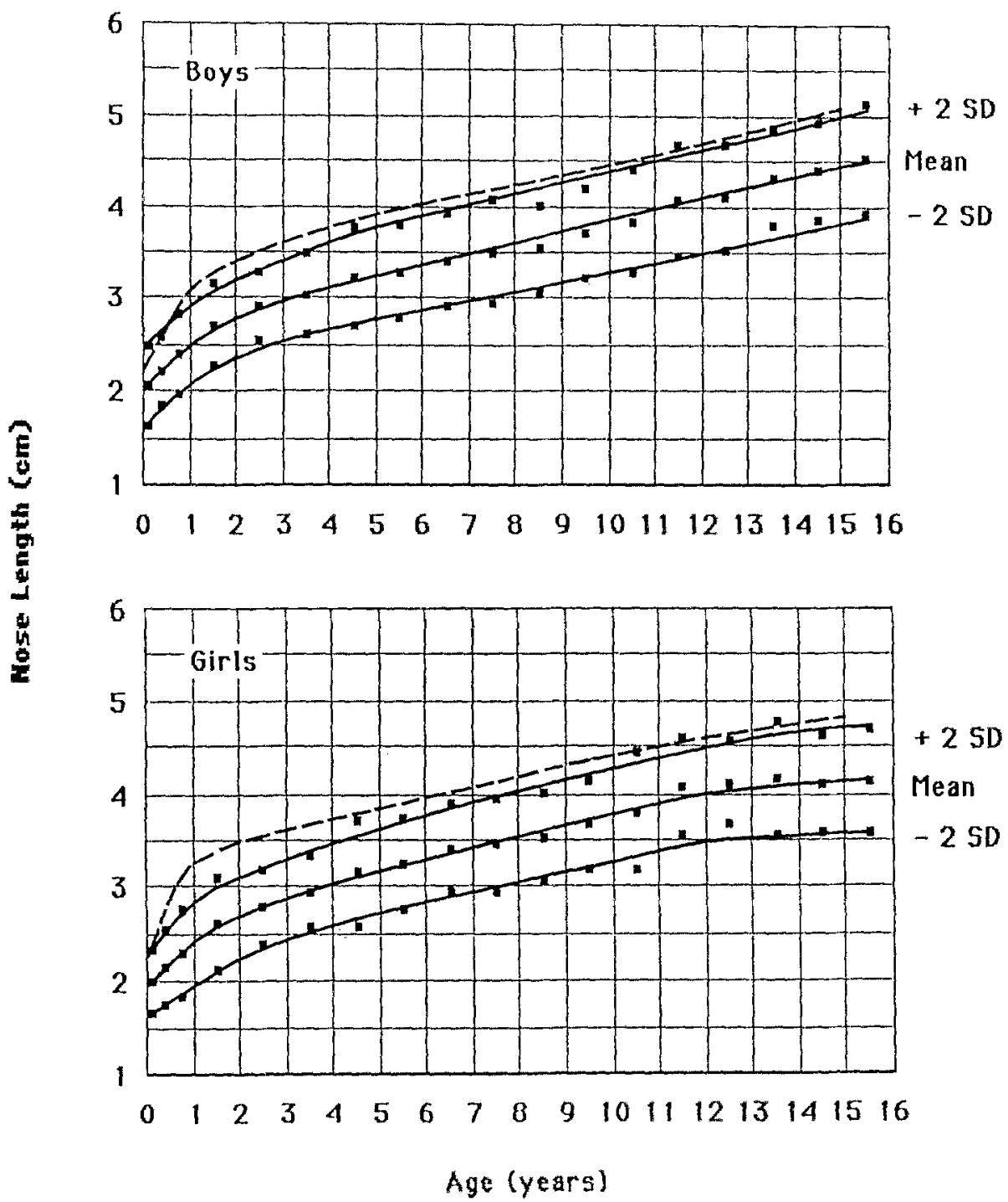




\section{Nose Breadth}

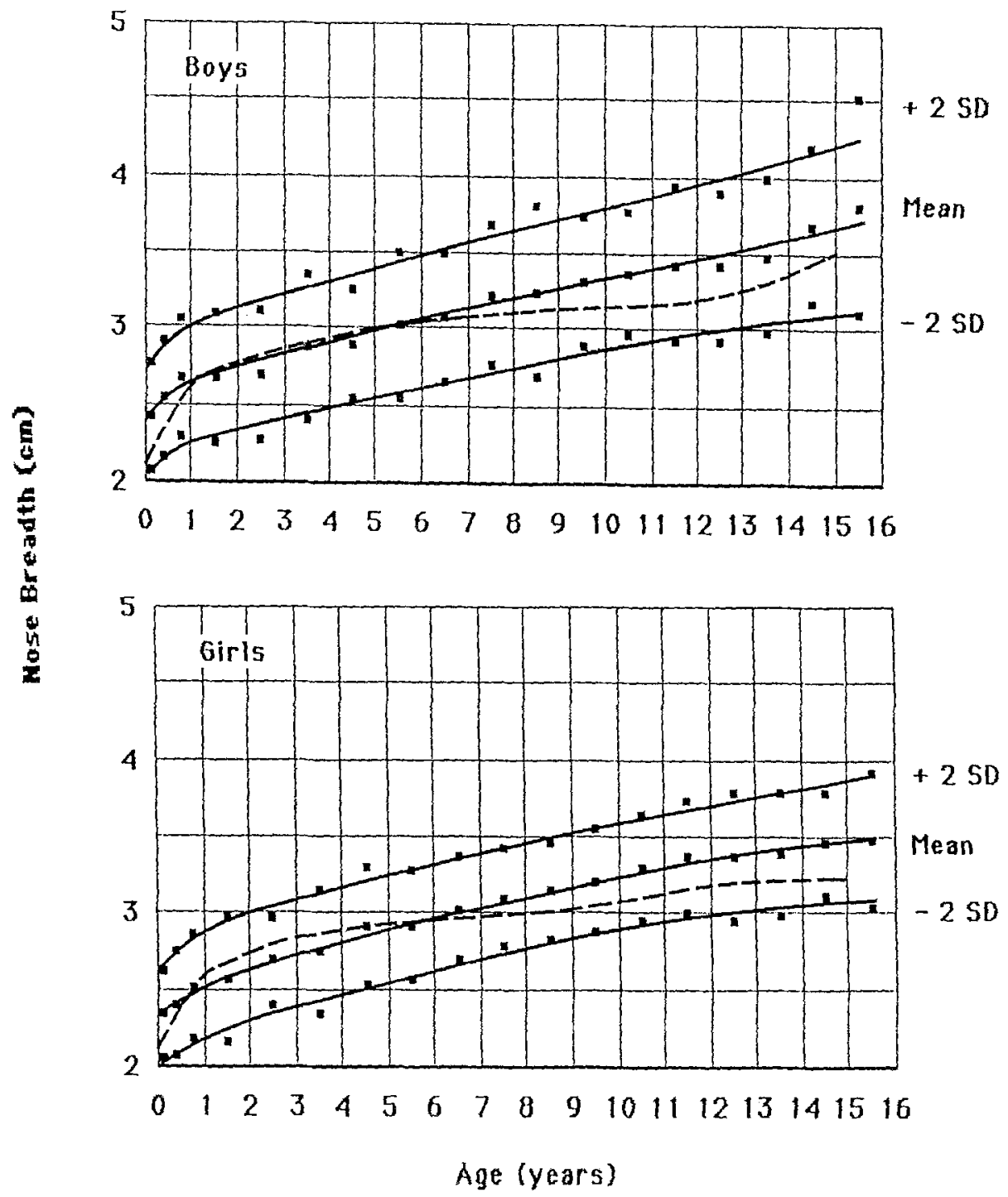

Vol. 33, No. 1, 1988 
12. Philtrum Length

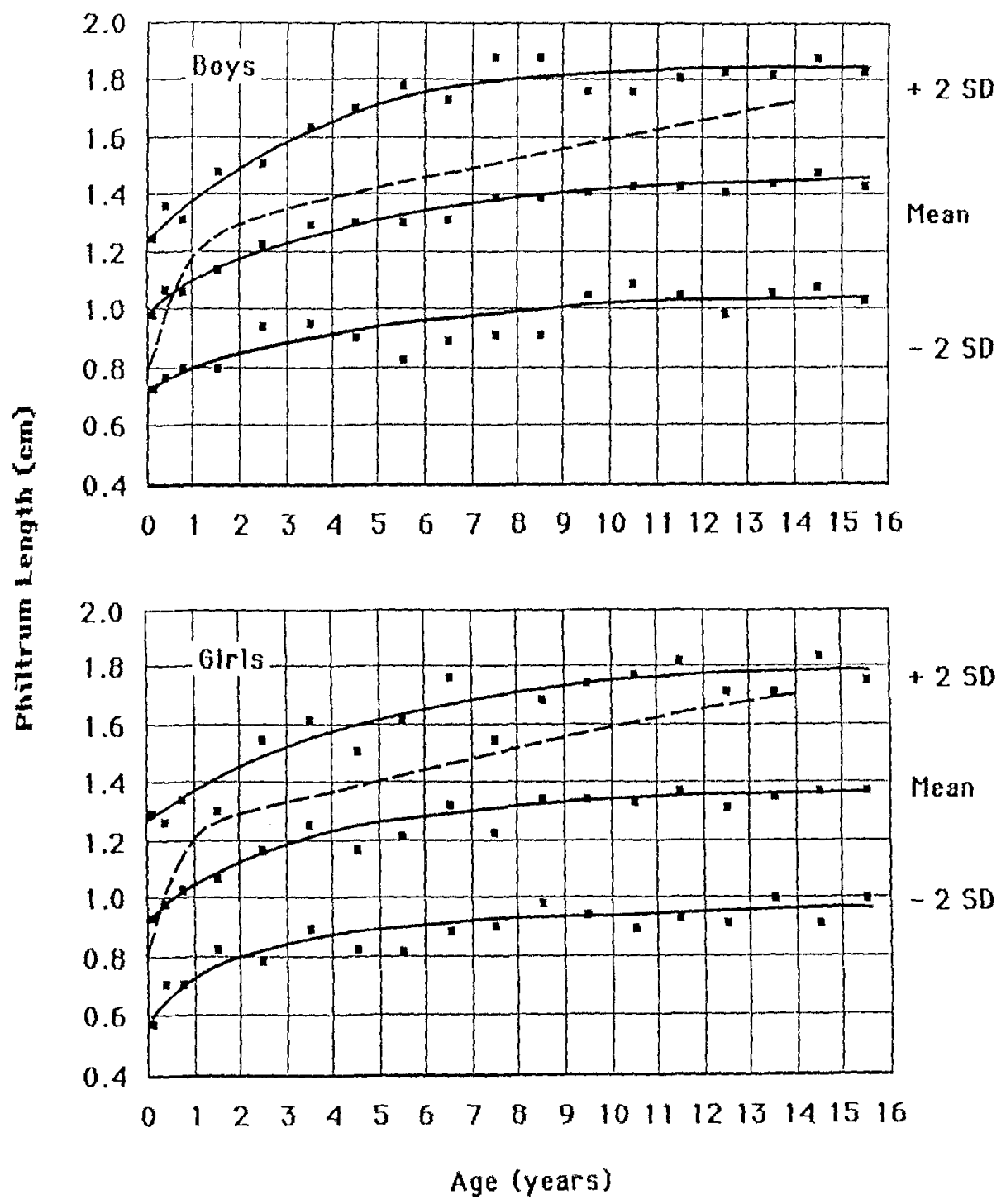




\section{Mouth Breadth}

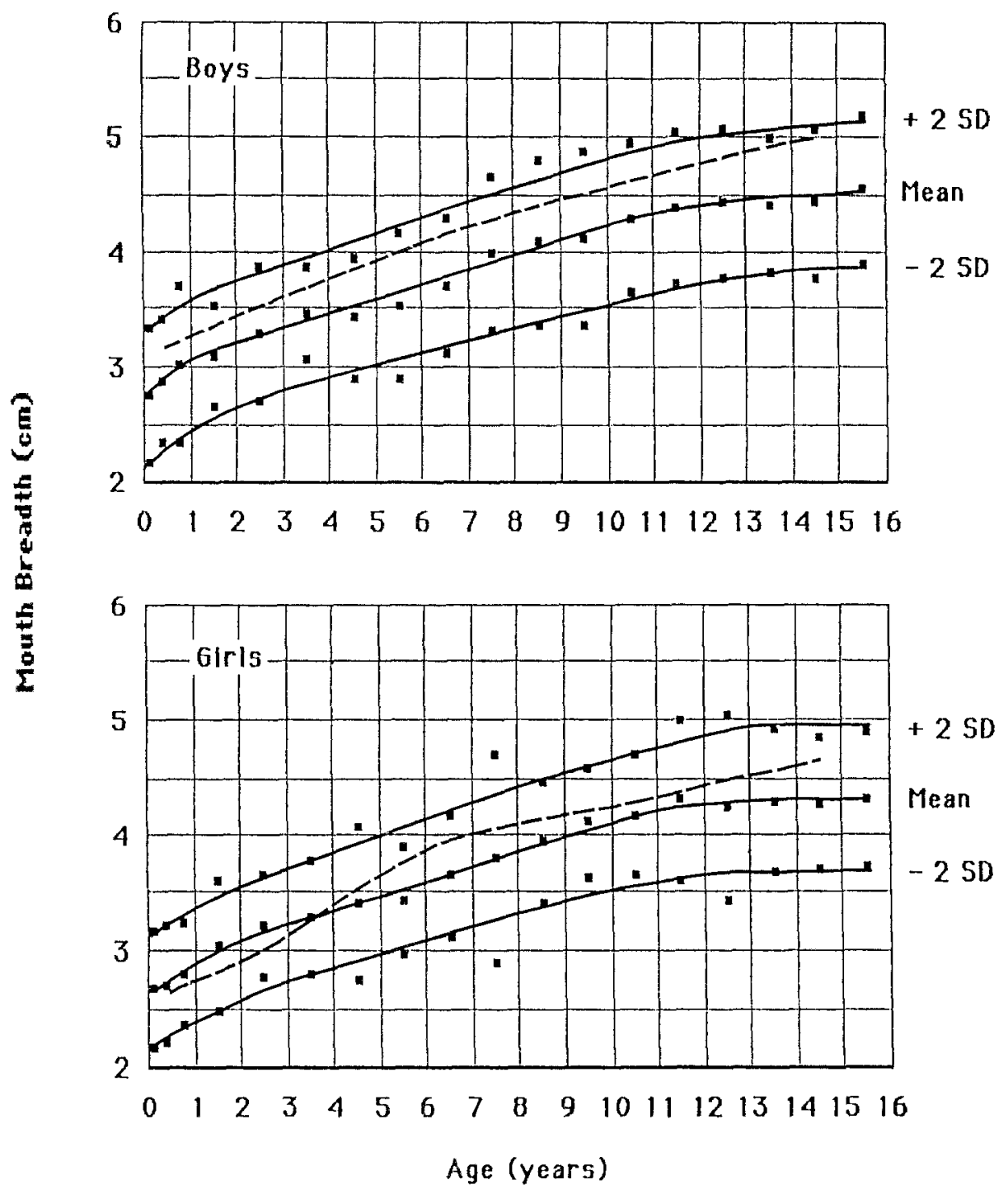




\section{Upper Lip Thickness}

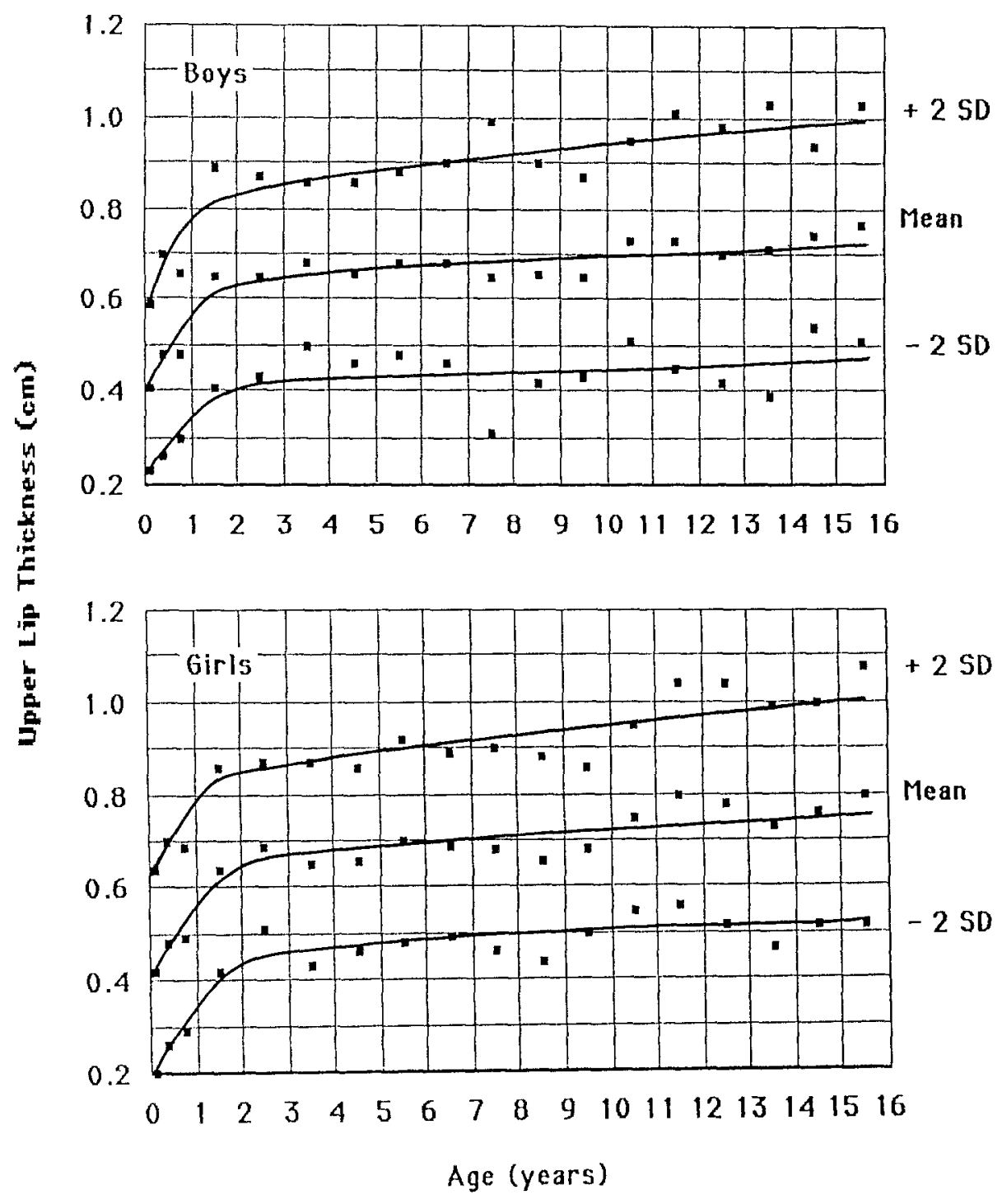


15. Lower Lip Thickness

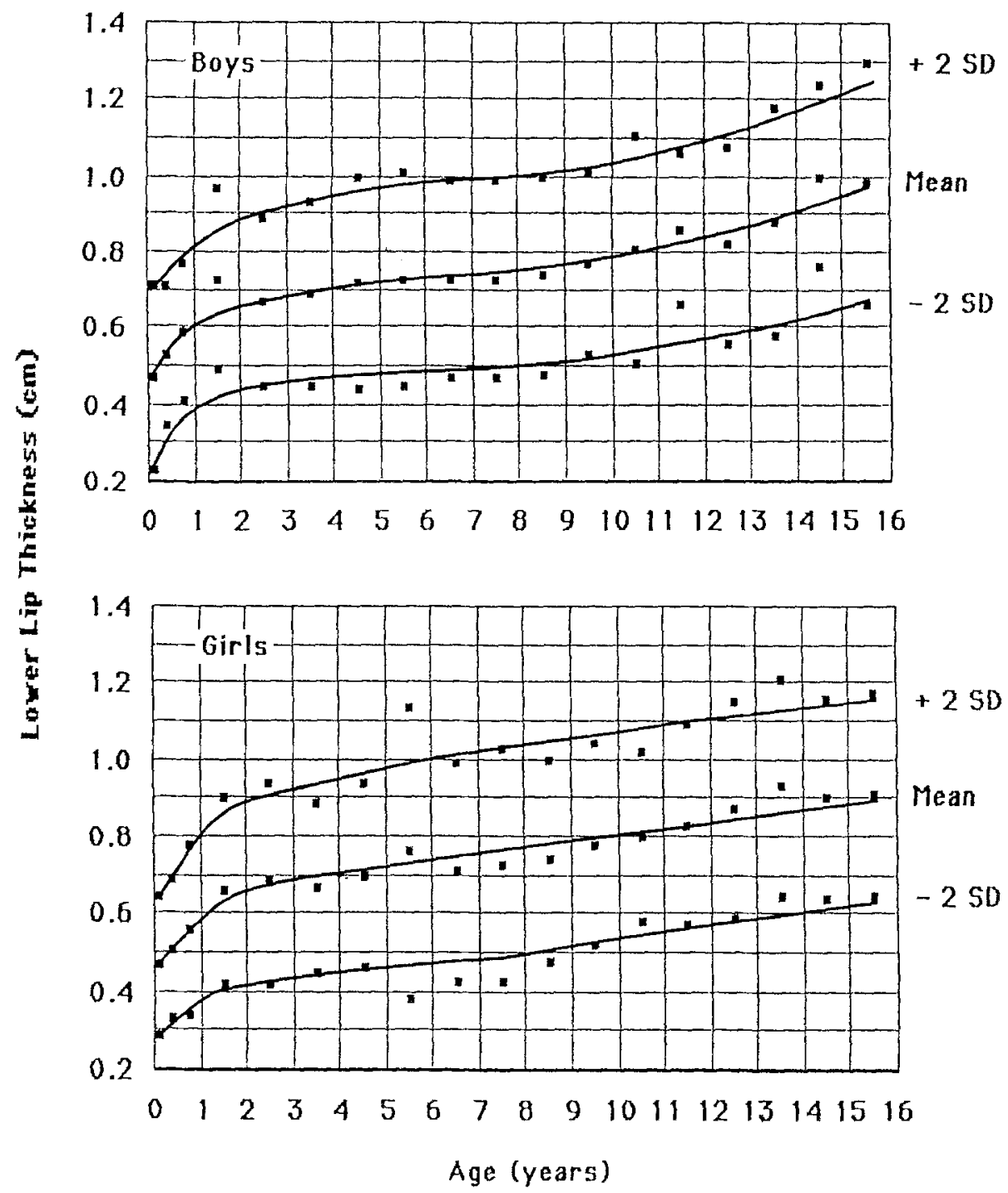

Vol. 33, No. ], 1988 
16. Ear Length

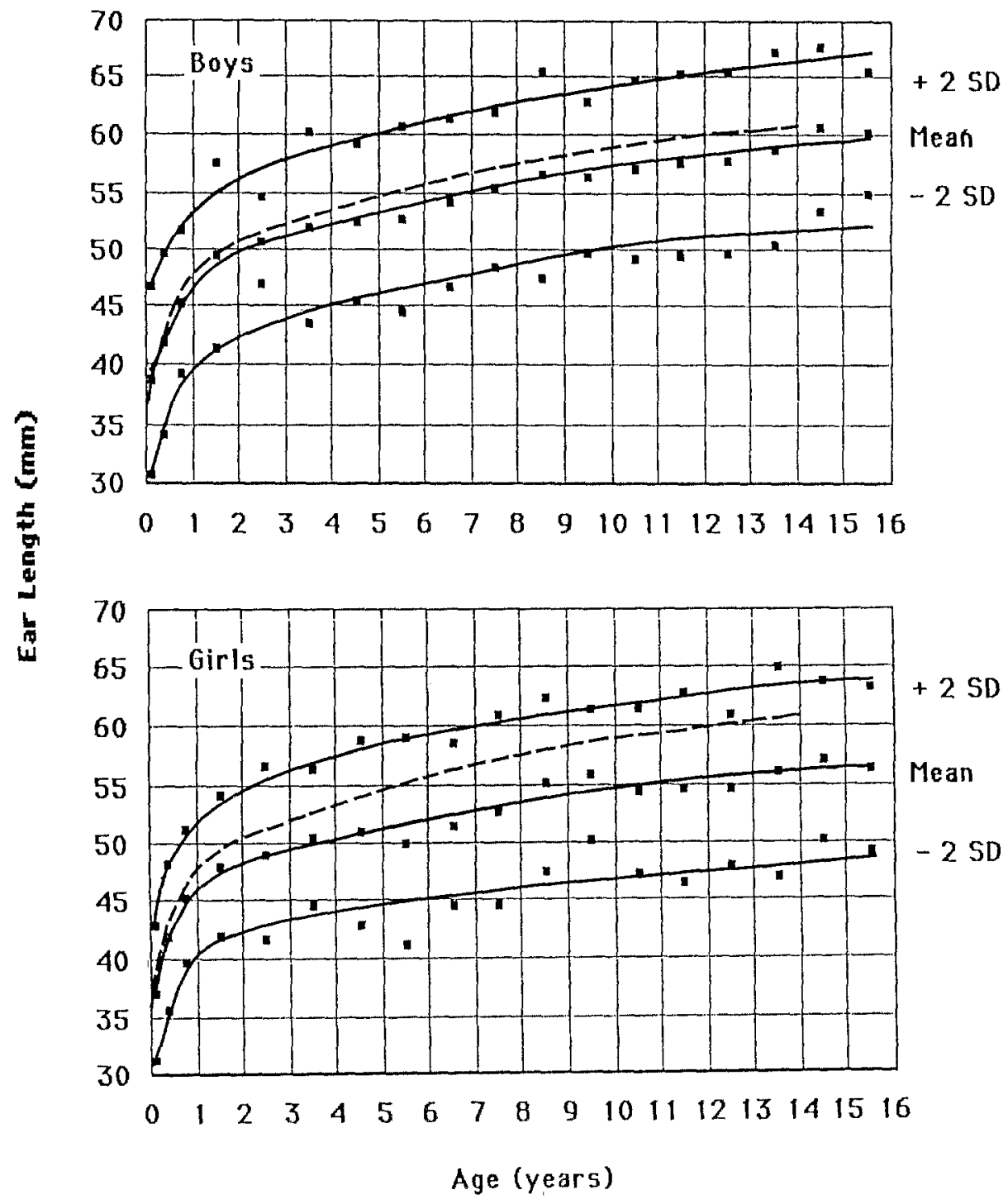


17. Palm Length

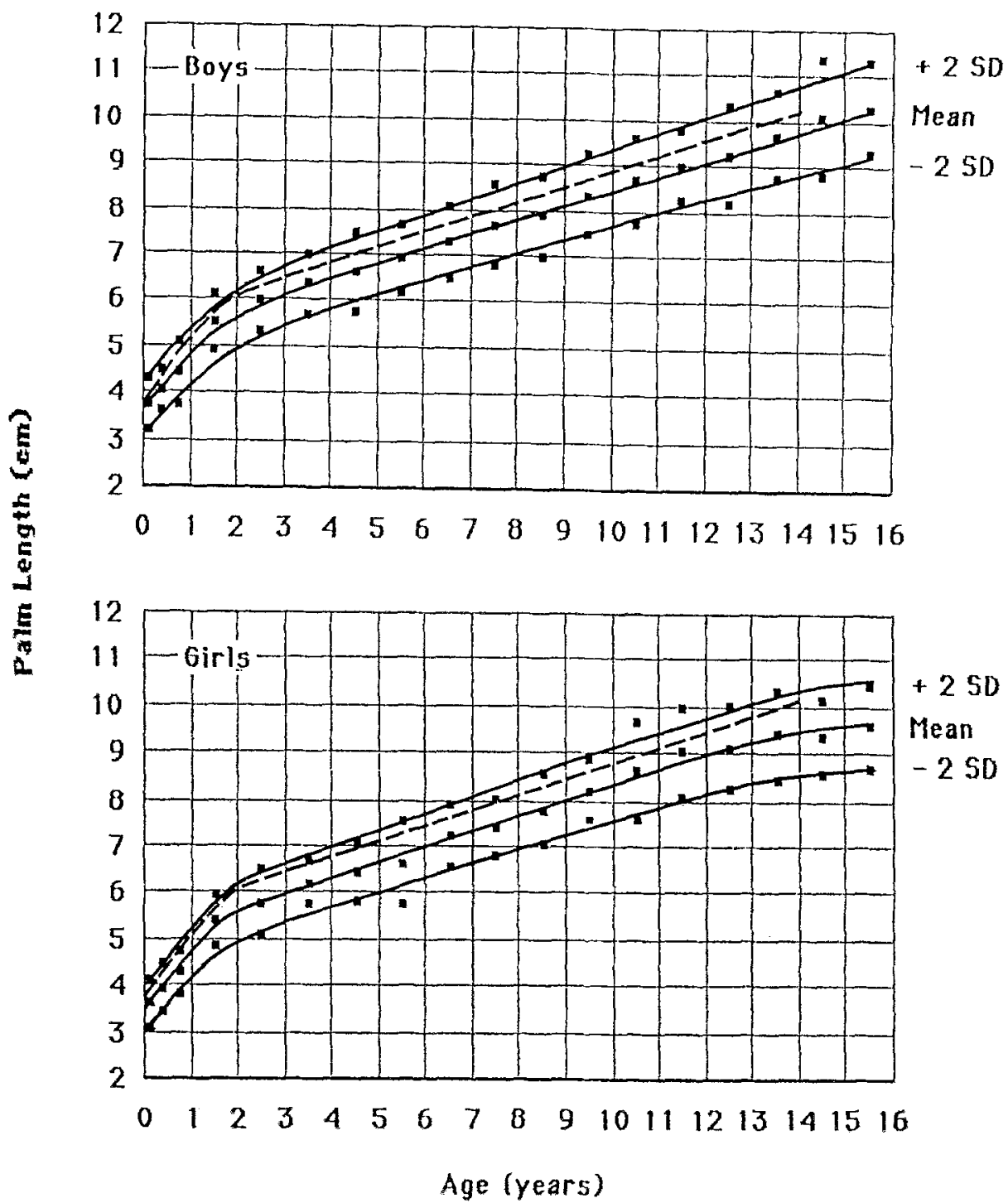


18. Middle Finger Length

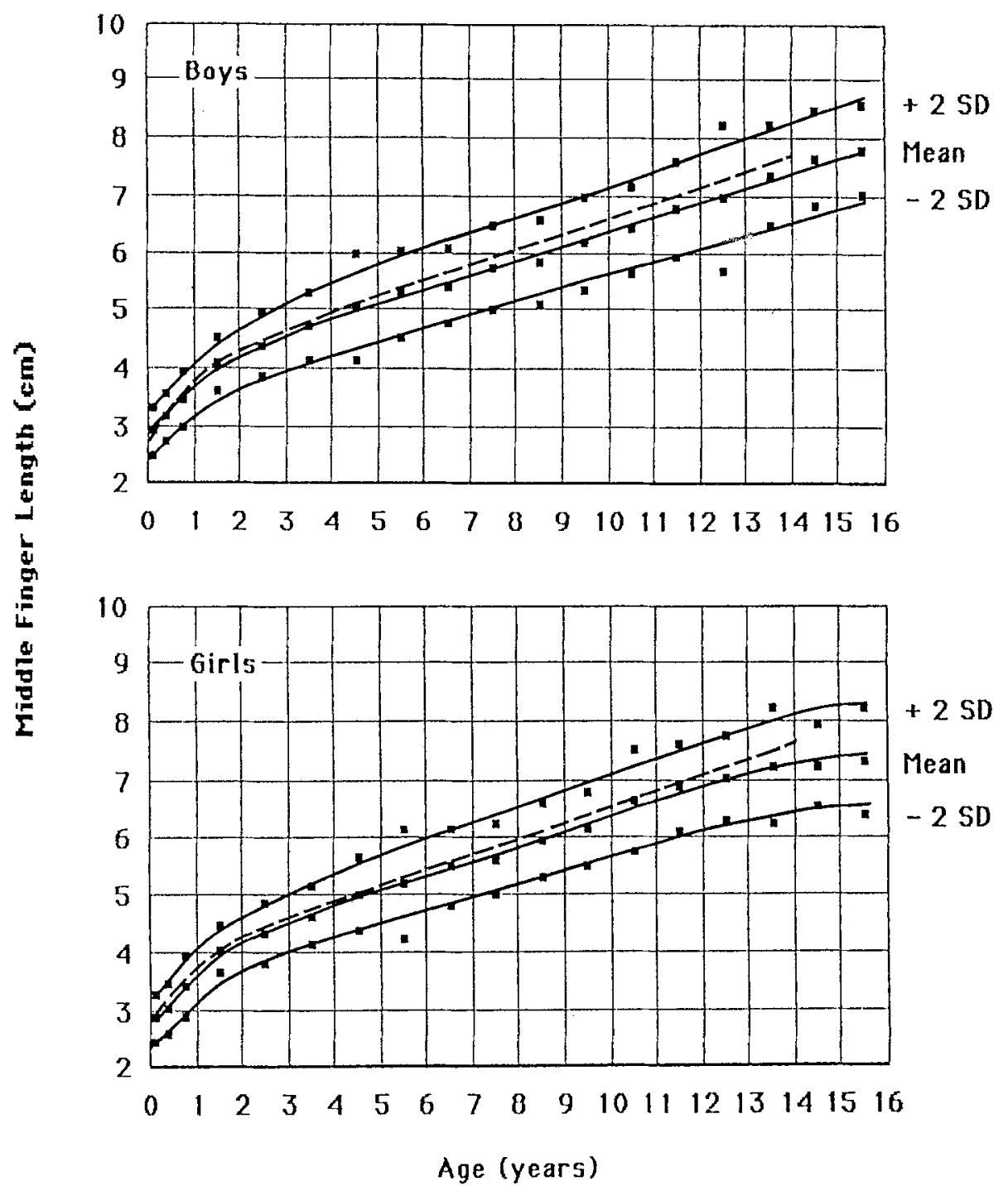


19. Fifth Finger Length

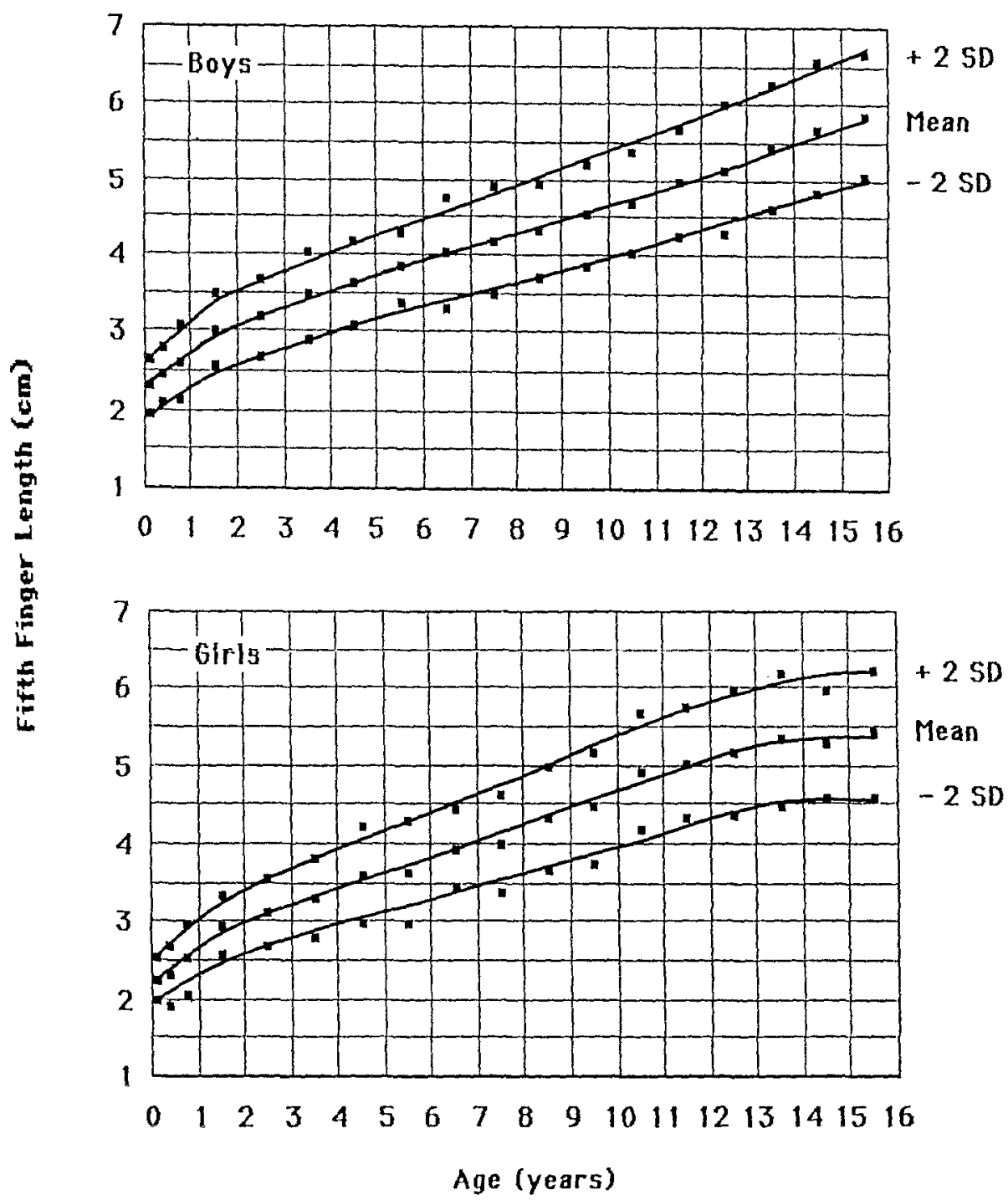

